\title{
EMISSÃO DE ÓXIDO NITROSO EM SOLOS COM DIFERENTES USOS E MANEJOS: UMA REVISÃO
}

\author{
Risely Ferraz de Almeida* \\ Emmanuel Rezende Naves** \\ Camila Haddad Silveira*** \\ Beno Wendling****
}

RESUMO: A agricultura é um dos principais setores que contribui para as emissões do óxido nitroso $\left(\mathrm{N}_{2} \mathrm{O}\right)$, sendo este gás um dos responsáveis pelo efeito estufa na atmosfera. A escolha do sistema de manejo e uso do solo utilizado na agricultura influencia no fluxo deste gás. O solo influencia no ciclo do nitrogênio através da disponibilidade de nitrato, temperatura, $\mathrm{pH}$, umidade, presença de $\mathrm{NH}_{4}^{+}$(íon de amônio) e conteúdo de compostos oxidantes que são utilizados como receptores de elétrons para a degradação da matéria orgânica do solo. Contudo, dentre as condições do solo citadas, o fator dominante que regula a formação e emissão do óxido nitroso é o espaço poroso ocupado por água (PPA) aliado à temperatura. Assim, solos aerados que apresentem um PPA de 35 a 60\% têm formação de óxido nitroso como um subproduto da nitrificação. E dentre os sistemas de cultivo utilizados no Brasil, o plantio direto - PD e o plantio convencional - PC ainda necessitam de maiores estudos para verificar a influência destes nos processos de produção e emissão de óxido nitroso dos solos. Diante do exposto, devem-se intensificar trabalhos e pesquisas realizadas com gases de efeito estufa - GEE, em diferentes manejos e usos do solo na agricultura, tendo a necessidade de alternativas ambientalmente corretas para mitigar a sua emissão para a atmosfera.

PALAVRAS-CHAVE: Agricultura; Atmosfera; Gases de Efeito Estufa; Nitrogênio.

\footnotetext{
"Doutoranda do Programa de Pós-graduação em Agronomia (Ciência do Solo) Universidade Estadual Paulista "Júlio de Mesquita Filho" (UNESP), campus Jaboticabal (SP), Brasil; E-mail: rizely@gmail.com

${ }^{* *}$ Mestre do Instituto de Ciências Agrárias, Universidade Federal de Uberlândia - UFU, campus Uberlândia (MG), Brasil.

*** Discente do curso de Engenharia Agronômica do Instituto de Ciências Agrárias, Universidade Federal de Uberlândia (UFU), campus Uberlândia (MG), Brasil.

**** Docente Doutor do Instituto de Ciências Agrárias, Universidade Federal de Uberlândia (UFU), campus Uberlândia (MG), Brasil.
} 


\title{
THE EMISSION OF NITROUS OXIDE IN SOILS WITH DIFFERENT USAGES AND MANAGEMENTS: A REVIEW
}

\begin{abstract}
Agriculture is one of the main emitter of nitrous oxide $\left(\mathrm{N}_{2} \mathrm{O}\right)$, one of the gases that cause the greenhouse effect in the atmosphere. The management system and soil usage in agriculture affects the gas flow. The soil affects the nitrogen cycle through the availability of nitrate, temperature, $\mathrm{pH}$, moisture, $\mathrm{NH}_{4}^{+}$(ammonia ion) and content of oxidizing compounds used as electron receivers for the degradation of the soil's organic matter. The dominating factor, within the above soil conditions, which regulate the formation and emission of nitrous oxide, is the porous space occupied by water (PSW) coupled to temperature. Aerated soil with PSW between 35 and $60 \%$ form nitrous oxide as a byproduct of nitrification. Within the culture systems used in Brazil, no-till farming and conventional farming still require further studies to verify their influence on the production process and emission of nitrous oxide from soils. Research works on greenhouse gases must be further undertaken at different managements and usages of soil in agriculture. Correct alternatives are required to lessen their emission into the atmosphere.
\end{abstract}

KEY WORDS: Agriculture; Atmosphere; Greenhouse Gases; Nitrogen.

\section{INTRODUÇÃO}

Nos países de clima tropical são crescentes as discussões a respeito das mudanças globais relacionadas à emissão de gases de efeito estufa - GEE. Em decorrência disso, observa-se um maior número de pesquisadores envolvidos e trabalhos publicados nesta área.

Os GEE podem ser tanto de origem antrópica como natural. Estudos recentes têm mostrado que há um aumento na concentração de dióxido de carbono $\left(\mathrm{CO}_{2}\right)$, gás metano $\left(\mathrm{CH}_{4}\right)$, óxido nitroso $\left(\mathrm{N}_{2} \mathrm{O}\right)$, óxidos de nitrogênio (NOx) e monóxido de carbono (CO) na atmosfera terrestre.

O setor da agropecuária, principalmente a agricultura, tem um papel importante no aumento desses gases no decorrer dos anos. Atualmente, em nível global, estima-se que 20\% dos GEE emitidos para a atmosfera sejam originários da atividade agrícola (JOHNSON et al., 2005). Dentre estes gases, o óxido nitroso tem menor concentração na atmosfera, assim como o $\mathrm{CH}_{4}$. No entanto, o poder de aquecimento 
(Global Warming Potential - GWP) do $\mathrm{N}_{2} \mathrm{O} \mathrm{CH}_{4}$ na atmosfera é muito superior, quando comparado com o $\mathrm{CO}_{2}$, que tem uma maior concentração na atmosfera.

Nos solos, o óxido nitroso pode ser produzido durante o processo microbiano de desnitrificação, quando bactérias anaeróbicas facultativas utilizam o nitrato $\left(\mathrm{NO}_{3}^{-}\right)$como aceptor final de elétrons em substituição ao oxigênio $\left(\mathrm{O}_{2}\right)$. Esse processo ocorre em condições de anoxia, sendo favorecido pela disponibilidade de carbono e pela presença de $\mathrm{NO}_{3}$, proveniente da mineralização da matéria orgânica do solo e da aplicação de fertilizantes minerais e orgânicos.

Objetiva-se, nesta revisão, expor os principais aspectos, do ponto de vista agrícola, da emissão do óxido nitroso $\left(\mathrm{N}_{2} \mathrm{O}\right)$, relacionando a emissão com as diferentes práticas de uso e manejo dos solos.

\section{DESENVOLVIMENTO}

\subsection{GASES DE EFEITO ESTUFA - GEE}

O efeito estufa é um mecanismo natural de aquecimento da atmosfera responsável por manter a temperatura média do planeta em níveis adequados para a existência dos seres vivos na biosfera. Esse fenômeno ocorre quando uma parte da radiação solar refletida pela superfície terrestre é absorvida por determinados gases, denominados de "gases de efeito estufa - GEE", presentes na atmosfera. Como consequência disso, a radiação infravermelha refletida pela Terra fica retida na baixa atmosfera resultando no aquecimento do planeta (ESCOBAR, 2008).

O efeito estufa é causado, principalmente, pela emissão de grandes quantidades e em velocidade acelerada de gases como o dióxido de carbono $\left(\mathrm{CO}_{2}\right)$, metano $\left(\mathrm{CH}_{4}\right)$, óxido nitroso $\left(\mathrm{N}_{2} \mathrm{O}\right)$ e o vapor d'água em relação à deposição dos mesmos na natureza (FROUZ et al., 2009).

Ambientes naturais, em estado conservado, são essencialmente emissores de gases de efeito estufa, entretanto a velocidade e a intensidade das emissões são menores, pois há um equilíbrio no ciclo biogeoquímico natural destes gases, com predomínio da deposição pela acumulação de biomassa (FROUZ et al., 2009).

Entre os GEE, o $\mathrm{CO}_{2}$ é o mais emitido para a atmosfera (cerca de $60 \%$ do 
total). Por sua vez, a quantidade de $\mathrm{CH}_{4}$ e $\mathrm{N}_{2} \mathrm{O}$ emitida para a atmosfera é bem menor quando comparada à de $\mathrm{CO}_{2}$, mas o potencial de aquecimento, respectivamente, é de 23 e 298 vezes maior que o $\mathrm{CO}_{2}$ (CERRI et al., 2007).

Nas últimas décadas, a atividade antrópica tem provocado uma série de alterações na paisagem terrestre e, mais recentemente, na atmosfera. $\mathrm{O}$ aumento da emissão de GEE e o consequente aquecimento global do planeta vêm acarretando a busca por estratégias que visem à redução das fontes desses gases (CARVALHO et al., 2010). Acredita-se que a agricultura é o setor que contribui com aproximadamente 20\% das emissões de GEE (JOHNSON et al., 2005).

Porém, há outros setores como energia, processos industriais, mudanças no uso da terra e florestas e tratamentos de resíduos, que são responsáveis por incrementar a emissão dos GEE no Brasil (MCTI, 2013).

De acordo com MCTI (2013), referente ao ano de 2010, tem-se as principais atividades poluidoras e suas respectivas percentagens de emissões de GEE em equivalente de $\mathrm{CO}_{2}$ : as atividades agropecuárias (35\%), o uso das terras e florestas (22\%), os setores relacionados à produção de energia (32\%), aos processos industriais (7\%) e aos tratamentos de resíduos (4\%). Entretanto, percebe-se o incremento na participação do setor agrícola nas emissões de GEE, sendo 20\% em 2005 e com 35\% de participação nas emissões em 2010.

\subsection{FONTES DE ÓXIDO NITROSO NA AGRICULTURA}

Conforme estudos de Cerri et al. (2009), a contribuição da agricultura na emissão de óxido nitroso para a atmosfera é de aproximadamente 87,2\% das emissões.

No Brasil, as emissões de óxido nitroso são oriundas principalmente dos solos, através da sua mudança de uso e manejo, representando cerca de $94 \%$ do total das emissões deste gás (CERRI; CERRI, 2007).

A emissão dos GEE do solo deve-se ao manejo inadequado que ocasiona vários processos degradativos com alterações dos processos físicos, químicos e biológicos (WASSMANN; LEK, 2004).

As principais fontes de liberação de óxido nitroso no solo são o uso de 
fertilizantes agrícolas nitrogenados, dejetos de animais, as queimas de combustíveis fósseis e processos naturais que ocorrem nos solos e nos oceanos (FORSTER et al., 2005).

\subsection{FORMAÇÃO DO ÓXIDO NITROSO NO SOLO}

Nos solos, a maior parte do óxido nitroso emitido advém de dois processos biológicos: a nitrificação e a desnitrificação, que fazem parte do ciclo de nitrogênio, assim como descrito por Zumft e Kroneck (2006) na Figura 1.

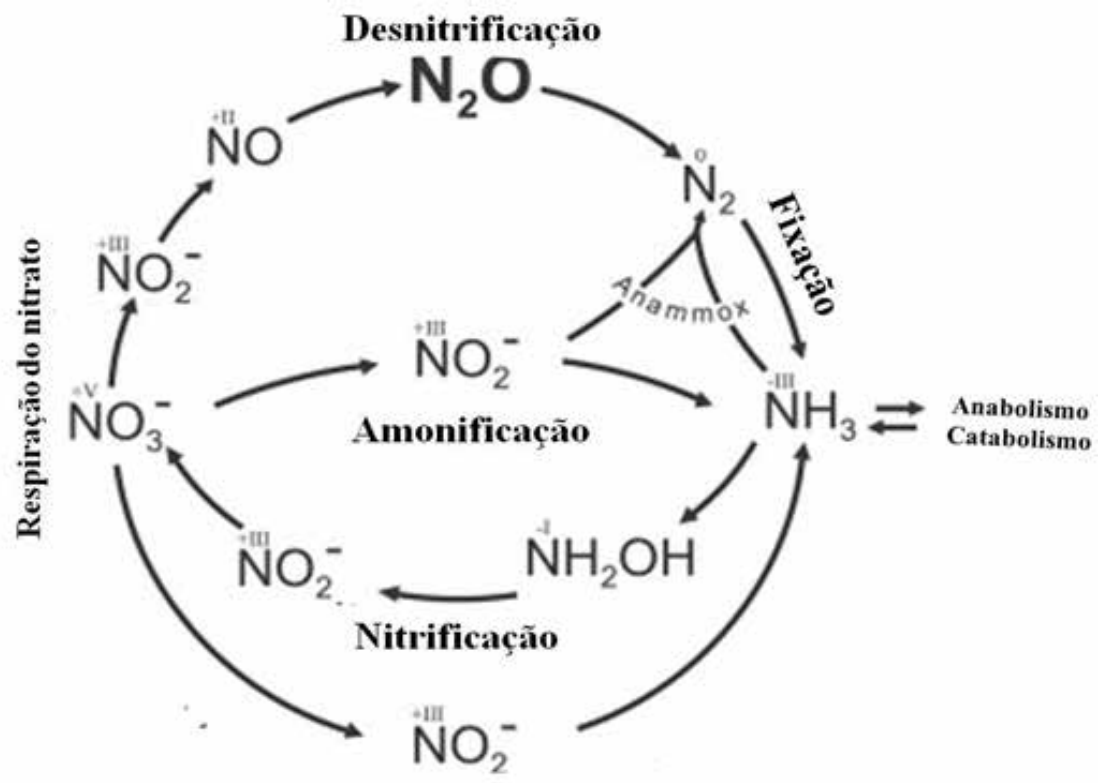

Assimilação do nitrato

Figura 1. Descrição do ciclo biogeoquímico do nitrogênio com a formação do óxido nitroso. Numerais romanos demonstram o estado de oxidação do átomo de nitrogênio. Figura adaptada, descrita por Zumft e Kroneck (2006).

A nitrificação é o processo de oxidação aeróbica de amônio a nitrito ou nitrato (DALAL et al., 2003). A oxidação aeróbica de amônio $\left(\mathrm{NH}_{4}^{+}\right)$a nitrato $\left(\mathrm{NO}_{3}{ }^{-}\right)$ é realizada por bactérias quimiautotróficas e ocorre em duas etapas: nitritação, em que o $\mathrm{NH}_{4}^{+}$é oxidado a nitrito $\left(\mathrm{NO}_{2}{ }^{-}\right)$por ação das Nitrossomonas sp., Nitrosospira sp. e Nitrococcus sp (MOREIRA; SIQUEIRA, 2006). 
As bactérias nitrossomonas e nitrosospira são as principais bactérias do solo e da água que oxidam amônia $\left(\mathrm{NH}_{3}\right)$ para nitrito $\left(\mathrm{NO}_{2}{ }^{-}\right)$. Enquanto que Nitrobacter é o principal gênero de bactérias que oxidam nitrito para nitrato $\left(\mathrm{NO}_{3}^{-}\right)$(HARRISON; WEBB, 2001; MOREIRA; SIQUEIRA, 2006).

A redução dissimilatória do nitrato a amônio se dá porque as bactérias fermentativas são capazes de reduzir nitrito a óxido nitroso, assim como a amônio, embora não sejam capazes de reduzir $\mathrm{N}_{2} \mathrm{O}$ a N $\mathrm{N}_{2}$. Este processo é favorecido quando há limitação de $\mathrm{NO}_{3}$ (STEVENS; LAUGHLIN, 1998).

De acordo com Varennes (2003), a reação de nitrificação ocorre em três reações e com a atividade das bactérias nitrossomonas e nitrobacter, conforme aponta a Figura 2 .

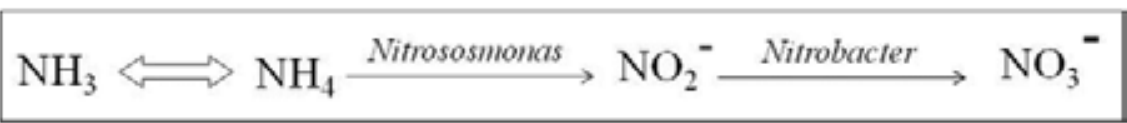

Figura 2. Equação de nitrificação demonstrando as atividades das bactérias Nitrossomonas e Nitrobacter, no ciclo biogeoquímico do nitrogênio nos solos. Fonte: Varennes (2003).

A desnitrificação é o processo de oxidação em que o $\mathrm{NO}^{3-}$ é transformado até $\mathrm{o} \mathrm{N}_{2}$, através da ação de bactérias anaeróbicas facultativas, as quais representam de 0,1 a 5\% da população total de bactérias no solo (MOREIRA; SIQUEIRA, 2006; VARENNES, 2003).

A desnitrificação é favorecida por condições de limitação de C (STEVENS; LAUGHLIN, 1998), sendo o óxido nitroso um produto da desnitrificação nas células microbianas que é emitido para o solo e a atmosfera (IPCC, 2007). A equação de desnitrificação é descrita por Varennes (2003) na Figura 3.

$$
\mathrm{NO}_{3}{ }^{-} \longrightarrow \mathrm{NO}_{2}^{-} \longrightarrow(\mathrm{NO}) \longrightarrow \mathrm{N}_{2} \mathrm{O} \uparrow \longrightarrow \mathrm{N}_{2} \mathrm{O} \uparrow
$$

Figura 3. Equação de desnitrificação no ciclo biogeoquímico do nitrogênio no solo, tendo a liberação do $\mathrm{N}_{2} \mathrm{O}$ (óxido nitroso) e o $\mathrm{N}_{2}$ (nitrogênio molecular) para o ambiente. Fonte: Varennes (2003). 
Também pode ocorrer a produção do óxido nitroso através da ação das bactérias fermentativas capazes de reduzir nitrito a óxido nitroso em condições estritamente anaeróbicas, $\mathrm{pH}$ elevado e grande quantidade de matéria orgânica facilmente decomponível que favorecem a redução dissimilatória de $\mathrm{NO}_{3}^{-}$a $\mathrm{NH}_{4}^{+}{ }^{+}$STEVENS; LAUGHLIN, 1998).

Além deste, uma pequena parte da produção e emissão do óxido nitroso dentro do ciclo do nitrogênio provém de um processo não biológico, como a decomposição química do nitrato (quimiodesnitrificação) e a decomposição química de hidroxilamina $\left[\mathrm{NH}_{2} \mathrm{OH}\right]$, produto intermediário na conversão de amônio $\left(\mathrm{NH}_{4}^{+}\right)$ a nitrito $\left(\mathrm{NO}_{2}^{-}\right)$e reação desta com o nitrito (STEVENS; LAUGHLIN, 1998; BREMMER, 2002; HATZENPICHLER, 2012).

Contudo, dentre os processos citados, a desnitrificação e a nitrificação são os mais significativos na emissão de óxido nitroso no solo (MOREIRA; SIQUEIRA, 2006).

\section{MÉTODOS PARA DETERMINAÇÃO DA EMISSÃO DE ÓXIDO NITROSO}

Há diferentes métodos que são utilizados para a avaliação da emissão do óxido nitroso do solo. As medidas diretas são realizadas através de campânulas posicionadas sobre a superfície do solo onde as amostras gasosas são coletadas manualmente, utilizando-se seringas (COSTA et al., 2006).

Essas campânulas estáticas são constituídas por uma base de metal parcialmente enterrada no solo (aproximadamente $5 \mathrm{~cm}$ de profundidade), e por uma tampa de PVC com uma cobertura para o acoplamento da seringa onde serão coletadas as amostras de óxido nitroso. Após as coletas, as amostras são encaminhadas para laboratório para serem analisadas em cromatógrafo (SIGNOR, 2010).

No cromatógrafo a análise desse gás pode ser através do detector de captura de elétrons (ECD), que é mais sensível e seletivo em uso atualmente, podendo detectar concentrações de até um picograma (10-9 g) deste gás nas amostras (GONÇALVES, 2002).

Os modelos FASSET e móvel-DNDC através de funções empíricas são capazes de estimar satisfatoriamente a emissão de óxido nitroso do solo com a aplicação 
de fertilizantes inorgânicos. No entanto, os dois modelos têm enfrentado vários desafios na modelagem das emissões de $\mathrm{N}_{2} \mathrm{O}$ do solo a partir de sistemas orgânicos de cultivo, especialmente aqueles que incluem culturas intercalares (CHIRINDA et al., 2011).

\subsection{CARACTERÍSTICAS FÍSICAS E QUÍMICAS DOS SOLOS QUE INFLUENCIAM NAS EMISSÕES DE ÓXIDO NITROSO}

Algumas práticas agrícolas favorecem as emissões gasosas a partir do solo, como desmatamento, queimada de biomassa, revolvimento do solo, implantação de culturas em áreas marginais (WASSMANN; VLEK, 2004).

Entretanto, os solos agrícolas por serem os principais emissores de óxido nitroso para a atmosfera, podem ser manipulados a fim de se afetar os processos de nitrificação e desnitrificação e, consequentemente, interferem na emissão dessa substância (SIGNOR; CERRI, 2013).

$\mathrm{O}$ aumento das perdas de óxido nitroso está associado ao aumento de umidade no solo, ocasionando aumento na quantidade de espaço poroso ocupado por água e consequente diminuição no $\mathrm{O}_{2}$ disponível (BRAGA et al., 2011).

Em solos com textura argilosa evidencia-se uma maior emissão de óxido nitroso, que pode estar associada à maior percentagem de poros preenchidos por água - PPA provocada pelo pequeno tamanho dos poros e altos conteúdos de umidade (SKIBA; BALL, 2002). Assim, nos solos, quando a difusividade dos gases é menor e a areação é limitada, o óxido nitroso será o produto dominante (DAVIDSON et al., 2000).

A disponibilidade de nitrato também é um fator essencial para que ocorram perdas de $\mathrm{N}$ na forma de óxido nitroso (CARMO et al., 2005), assim como a temperatura, o pH do solo, a presença de $\mathrm{NH}_{4}^{+}$(JANTALIA et al., 2006). Além deste, também pode-se citar o potencial redox e o conteúdo de compostos oxidantes no solo $\left(\mathrm{O}_{2}\right.$, $\mathrm{NO}_{3} ; \mathrm{Mn}^{4+}, \mathrm{Fe}^{3+}, \mathrm{SO}_{4}^{2}-\mathrm{e} \mathrm{CO}_{2}$ ) que são utilizados como receptores de elétrons para a degradação da matéria orgânica (YU et al., 2001).

Dentre as condições do solo citadas, o fator dominante que regula a formação de óxido nitroso nos solos é o espaço poroso ocupado por água (PPA) aliado à 
temperatura. Assim, solos aerados que apresentem um PPA de 35 a 60\% têm formação de óxido nitroso como um subproduto da nitrificação. Já em PPA acima de 70\%, são percebidas condições que facilitam as reações de desnitrificação ocorrendo uma maior emissão deste gás (JANTALIA et al., 2006).

\subsection{EMISSÕES DE ÓXIDO NITROSO EM DIFERENTES PRÁTICAS DE USOS E MA- NEJOS DOS SOLOS}

Em solos com vegetação de Cerrado natural, a emissão de óxido nitroso é muito baixa, chegando a ser quase nula em certos períodos do ano, entretanto, maiores emissões são registradas no início do período chuvoso seguido por decréscimo na emissão de óxido nitroso nos meses seguintes. A causa dessa variação sazonal se deve ao acúmulo de $\mathrm{N}$ orgânico no solo durante o período seco e às altas taxas de nitrificação possibilitadas no início das águas (CARDOSO et al., 2001).

Solos sob plantio direto - PD têm geralmente maior umidade, carbono orgânico - CO e populações microbianas mais concentradas perto da superfície do solo do que solos sob plantio convencional - PC, condições que favorecem a produção de óxido nitroso (LEMKE et al., 2004).

A maior disponibilidade de carbono orgânico - CO solúvel e a atividade desnitrificadora relativamente superior no solo sob PD podem ser responsáveis pelas maiores emissões de $\mathrm{N}_{2} \mathrm{O}$ (LIU et al., 2007). Além destes, a maior emissão de óxido nitroso a partir de solos sob $\mathrm{PD}$, pode ser devido à compactação, porosidade reduzida, aumento da PPA e aumento da desnitrificação (GOMES, 2006; LIU et al., 2007).

Em sistema de rotação de culturas com aveia/soja/aveia + ervilhaca/ milho/ nabo/trigo/soja, conduzida sob PC e PD após a colheita da soja (PCs e PDs), e no sistema de rotação de culturas aveia + ervilhaca/milho/nabo/trigo/soja/aveia/soja em PD após a colheita do milho e durante a cultura de nabo (PDm), pode-se constatar maiores emissões de óxido nitroso do solo sob PD com resíduos de soja (PDs), apresentando tendência de valores mais elevados no começo das avaliações. No solo sob PDs, verifica-se que as emissões alcançaram valores bem maiores do que no PCs (ESCOBAR, 2008).

No cultivo da soja as maiores emissões de óxido nitroso acontecem no período compreendido entre 20 dias antes e 20 dias após a colheita. Estes elevados 
níveis de emissões aparentemente estão relacionados às maiores concentrações de C lábil, produto da senescência nodular, que é consumido e utilizado como substrato elementar para o crescimento das populações microbianas, favorecendo desta maneira a totalidade de microrganismos e especificamente os nitrificadores e desnitrificadores do solo (CIAMPITTI; CIARLO; CONTI, 2005).

Além dessas condições, quando a cultura da soja encontra-se na região de Cerrado e em estação chuvosa, a emissão de $\mathrm{N}_{2} \mathrm{O}$ é maior do que em ambientes de pastagem e Cerrado natural (SAMINÊZ, 1999).

Portanto, o uso de leguminosas fixadoras de $\mathrm{N}_{2}$ nos sistemas agrícolas aumenta os teores de nitrogênio no solo e as emissões de NO e óxido nitroso (SAMINÊZ, 1999; GOMES et al., 2009; CHAVEZ, 2011).

Em sistemas de cultivos acumuladores de materiais orgânicos, sobretudo em sistemas de cultivo direto, aumenta-se a disponibilidade de fontes de carbono no solo. Entretanto, a rápida decomposição da matéria orgânica pode ocasionar anaerobiose no solo, devido ao consumo de oxigênio pelos microrganismos do solo (VELTHOF; KUIKMAN; OENEMA, 2002).

A anaerobiose proporcionaria condições satisfatórias para os organismos desnitrificantes, ocasionando a conversão do $\mathrm{NO}_{3}$ a óxido nitroso e sua posterior volatilização para a atmosfera (PIMENTEL, 2014).

Porém, os fluxos de óxido nitroso parecem ter sua expressão relacionada com a disponibilidade primeiramente de nitrato no solo do que com o carbono do solo, pois, a desnitrificação demostrou ser improvável de acontecer na ausência de íons nitrato e de ambiente anaeróbico (BRANCO, 1975), apesar da grande correlação entre o potencial de desnitrificação do solo e o suprimento de $\mathrm{N}$ e C encontrados em condições laboratoriais (CARMO et al., 2005).

Em área com o sistema de integração Lavoura-Pecuária - ILP ocorre um maior adensamento das camadas do solo favorecendo a emissão do óxido nitroso nos períodos de chuva onde elevam a umidade e o processo de desnitrificação, que é a maior fonte de emissão deste gás (PIVA et al., 2009).

Comparando os sistemas de ILP com pastagem permanente, foram encontrados maiores valores de emissão de óxido nitroso, cerca de duas vezes, na área correspondente à ILP somando-se valores de emissão na estação chuvosa e seca, 
resultantes da deposição de fezes e urinas de bovinos nas pastagens (TOMAZI et al., 2013). Segundo estes autores, isto se deve, talvez, ao sistema de ILP ser cultivado a cada dois anos com aveia/soja, o que disponibilizaria maiores quantidades de $\mathrm{N} \mathrm{e}$ $\mathrm{C}$ para atividade microbiológica, enquanto que a área correspondente à pastagem permanente encontrava-se há 16 anos sem fertilização.

Em comparação entre os sistemas ILPF (cultivo de soja, Eucalyptus grandis e pastagem), ILP (cultivo de soja e pastagem) e vegetação nativa (Cerrado), observaram-se diferenças. $\mathrm{Na}$ área de Cerrado observaram-se valores negativos de emissão de $\mathrm{N}_{2} 0$, representando assim uma fonte de consumo deste elemento. Já o sistema ILPF apresentou menores taxas de emissão de óxido nitroso em relação ao sistema ILP, constituindo-se uma melhor alternativa dentre os sistemas de cultivo como de baixa emissão de óxido nítrico (OLIVEIRA et al., 2012).

Em áreas de Mata Atlântica, nas quais ocorre a substituição de pastagem de baixa produtividade por reflorestamento com eucalipto e floresta secundária, as emissões de óxido nitroso são maiores na área com a floresta, onde também foram encontrados os maiores teores de $\mathrm{NO}_{3}^{-}$. A qualidade dos resíduos desta área influencia a incorporação de matéria orgânica, pela menor relação $\mathrm{C} / \mathrm{N}$ observada, o que estimula a mineralização de matéria orgânica do solo - MOS e resulta em maiores teores de nitrato. As emissões de óxido nitroso e os teores de $\mathrm{NO}_{3}$, na área com eucalipto, foram intermediários às áreas de mata e de pastagem (COUTINHO et al., 2010).

O uso de dejetos líquidos de suínos e de bovinos em PD com milho aumenta a emissão de óxido nitroso, principalmente nos primeiros 15 dias após a aplicação dos materiais orgânicos (DENEGA, 2009). Isso ocorre pois a urina e o esterco depositados sobre o solo promovem uma alta concentração localizada de $\mathrm{N} \mathrm{e}$ $\mathrm{C}$ prontamente disponível que estimula a atividade dos microrganismos envolvidos nos processos de transformação do N no solo (CARTER, 2007).

Os resíduos animais, apesar de constituírem excelentes fontes de nutrientes para os cultivos, podem contribuir para a liberação de gases de efeito estufa. No Brasil, somente o manejo dos dejetos animais foram responsáveis por 4,9\% das emissões de dióxido de carbono equivalente do setor agropecuário em 2010 (MCTI, 2013). 
Este parece ser o caso, principalmente, da urina de bovinos. De acordo com Tomazi et al. (2013), em estudo com a aplicação de doses crescentes de fezes e urina de bovinos nos sistemas de integração lavoura-pecuária e pastagem permanente, encontraram resultados que apontam que somente a urina teve a capacidade de aumentar as emissões de óxido nitroso e as taxas de emissão eram doses dependentes.

Em pastagens com a aplicação de resíduos líquidos oriundos da criação de ovinos ocorre um aumento na emissão de óxido nitroso do solo, em comparação com a aplicação de esterco e o tratamento sem aplicação de dejetos. Os fluxos de $\mathrm{N}_{2} \mathrm{O}$ variam de 4 a $353 \mu \mathrm{g} \mathrm{N}-\mathrm{N}_{2} \mathrm{O} \mathrm{m}^{-2} \mathrm{~h}^{-1}$, sendo as maiores emissões correspondentes às maiores quantidades de urina aplicadas. $\mathrm{O}$ efeito da urina no aumento das emissões de óxido nitroso é observado até os 23 dias após a aplicação (MAGIERO et al., 2011).

A adição de fertilizante amoniacal aos sistemas agrícolas é uma fonte antrópica de óxido nitroso, derivada da nitrificação. Já as emissões de $\mathrm{N}_{2} \mathrm{O}$ provenientes da desnitrificação são altamente variáveis porque o $\mathrm{N}_{2} \mathrm{O}$ não é o produto terminal da desnitrificação, sendo que as emissões de $\mathrm{N}_{2} \mathrm{O}$ são, em geral, maiores em ambientes úmidos (DUXBURY, 1995; DAVIDSON et al., 2000).

Carvalho et al. (2011), avaliando a emissão de óxido nitroso em cultivo de arroz com a adição de adubos nitrogenados, encontraram um efeito significativo da adubação sobre a emissão de óxido nitroso, constatando que com a fertilização ocorre um aumento na emissão cerca de seis a oito vezes quando comparado com os tratamentos sem nitrogênio.

Com a aplicação da ureia após o terceiro dia em cobertura nos solos são proporcionados valores elevados na emissão de óxido nitroso. No entanto, depois de cinco dias da fertilização a emissão de NO é semelhante à de solo sob vegetação nativa de Cerrado (CARVALHO et al., 2008).

Comparando-se a aplicação de fertilizantes nitrogenados (ureia) e a aplicação de vinhoto na perda de $\mathrm{N}$ na forma de óxido nitroso em área de lavoura de cana-de-açúcar, constata-se que após 168 horas da aplicação das fontes de $\mathrm{N}$ há um aumento significativo na emissão. Na aplicação de vinhoto é apresentada uma maior emissão de óxido nitroso, enquanto que os tratamentos com as doses de 60 e 120 $\mathrm{kg}$ de $\mathrm{N} \mathrm{ha}^{-1}$ apresentaram perdas semelhantes àquela sem adição de fertilizantes (BRAGA et al., 2011). 
Fertilizantes nitrogenados adicionados nos sistemas de cultivo são rapidamente transformados pelos organismos do solo em nitrato $\left(\mathrm{NO}_{3}{ }^{-}\right)$. Este elemento possui dinâmica muito intensa no solo, sendo facilmente lixiviado pelo perfil do solo ou desnitrificado para a forma gasosa (óxido nitroso) e volatilizada do solo. Apesar da adubação nitrogenada adicionada aos solos representar uma importante fonte de emissão de óxido nitroso, ela se constitui imprescindível para a alta qualidade e quantidade da produção agrícola. Entretanto, existem tecnologias que podem amenizar as emissões de óxido nitroso, sendo uma delas os inibidores da nitrificação e de urease (FRYE, 2005).

Os inibidores de nitrificação agem mantendo o nitrogênio do solo na forma de amônio $\left(\mathrm{NH}_{4}^{+}\right)$por mais tempo, evitando assim tanto a lixiviação como a desnitrificação. A lixiviação e a desnitrifcação são impedidas, respectivamente, pela ligação do amônio na ctc do solo, e pela redução da disponibilidade de substrato necessário $\left(\mathrm{NO}_{3}^{-}\right)$para os organismos desnitrificantes (FRYE, 2005).

Logo, a utilização de inibidores de nitrificação e de urease pode mitigar a produção de $\mathrm{N}_{2} \mathrm{O}$. Estudos mostraram que a aplicação de inibidores de nitrificação e uréase, mesmo quando aplicados com a maior dose de urina de ovinos (fonte emissora de óxido nítrico), foram eficientes em diminuir a emissão de $\mathrm{N}_{2} \mathrm{O}$ em um argissolo em área de iLPF, em comparação com a mesma dose de urina mas sem os inibidores (MAGIEIRO, 2013).

Em um experimento em solo incubado com diferentes doses de nitrogênio mineral $\left(\left(\mathrm{NH}_{4}\right)_{2} \mathrm{SO}_{4}\right)$ e de palha de cana-de-açúcar, com a presença ou não de inibidor de nitrificação, mostrou-se que a adubação de nitrogenada independente da quantidade de palhada no solo aumentou a emissão de $\mathrm{N}_{2} \mathrm{O}$, e que o emprego do inibidor de nitrificação (dicianodiamida) demonstrou capacidade em reduzir em 60\% a liberação de óxido nitroso em comparação com os tratamentos com adubação nitrogenada e sem adição do inibidor (VARGAS et al., 2012).

Estes mesmos autores concluíram que a palhada da cana aumenta a liberação de $\mathrm{N}_{2} \mathrm{O}$ quando ocorre a aplicação do nitrogênio mineral e que a aplicação de inibidores de nitrificação seriam eficientes em reduzir a liberação deste gás. 


\section{CONSIDERAÇÕES FINAIS}

A agricultura é um dos setores que contribui para a emissão do óxido nitroso, sendo este um dos GEE que possui um alto poder de aquecimento global, quando comparado com o $\mathrm{CO}_{2}$. A escolha do sistema de manejo e uso do solo utilizado influencia na emissão do óxido nitroso, sendo que o acúmulo de $\mathrm{N}$ orgânico/ inorgânico no solo aumenta esta emissão. Adubações nitrogenadas, tanto orgânicas quanto inorgânicas, aumentam o fluxo deste gás do solo. O PD e o PC são manejos que requerem maiores estudos para verificar ainda a influência destes no processo de produção e emissão de óxido nitroso nos solos. Diante do exposto, devem-se intensificar trabalhos e pesquisas realizadas com GEE em diferentes manejos e usos do solo na agricultura, pois há a necessidade de alternativas ambientalmente corretas para mitigar a emissão de óxido nitroso do solo para a atmosfera.

\section{AGRADECIMENTOS}

Os autores agradecem à Fundação de Amparo à Pesquisa do Estado de Minas Gerais (FAPEMIG) e à Coordenação de Aperfeiçoamento de Pessoal de Nível Superior (CAPES) pelo apoio e incentivo à pesquisa.

\section{REFERÊNCIAS}

BRAGA, D.M.; COSTA, M.K.L.; PONTES, T.L.; ALVES, B.J.R.; SHIGAKI, F. Volatilização de amônia e emissão de óxido nitroso em função da adubação com uréia e vinhoto em cana-de-açúcar. Uberlândia/MG, 2011. In: CONGRESSO BRASILEIRO DE CIÊNCIA DO SOLO - CBCS, 33., 2011, Uberlândia/MG, Anais... Uberlândia: Universidade Federal de Uberlândia/UFU, 2011, v. 1, p. 1-3.

BRANCO, S. A. Análise de alguns aspectos e soluções prováveis para o Lago Paranoá. Rev. DAE, São Paulo, v. 26, n. 109, p. 38-45, 1975.

BREMMER, J.M.; WEIL, R.R. The nature and properties of soils. $13^{\text {th }}$ ed. Prentice Hall: Upper Saddle River, 2002. 960 p. 
CARDOSO, A.N.; SAMINÊZ, T.C.; VARGAS, M.A. Fluxos de gases-traços de efeito estufa na interface solo/atmosfera em solos de Cerrado. Planaltina/DF. Embrapa Cerrados, 2001. 33f. (Embrapa Cerrado. Boletim de Pesquisa e Desenvolvimento, 17).

CARMO, J.B.; ANDRADE, C.A.; CERRI, C.C.; PICCOLO, M.C. Disponibilidade de nitrogênio e fluxos de $\mathrm{N} 2 \mathrm{O}$ a partir de solo sob pastagem após aplicação de herbicida. Revista Brasileira de Ciência do Solo, v. 29, p. 735-746, 2005.

CARTER, M.S. Contribution of nitrification and denitrification to N2O emissions from urine patches. Soil Biology and Biochemistry, v. 3, n. 9, p. 2091-2102, 2007.

CARVALHO, A.M.; BUSTAMANTE, M.M.C.; KOZOVITS, A.R.; MIRANDA, L.M.; CARVALHO, J.L.N.; AVANZI, J.C.; SILVA, M.L.N.; MELLO, C.R.; CERRI, C.E.P. Potencial de sequestro de carbono em diferentes biomas do Brasil. Revista Brasileira de Ciência do Solo, v. 34, p. 277-289, 2010.

CARVALHO, A.M.; SOUZA, L.L.P.; ALVES, B.J.R.; MIRANDA, L.N. Aplicações de diferentes fertilizantes nitrogenadas: Emissões de óxido nitroso em latossolo sob feijão e milho. Brasília/DF. In: SIMPÓSIO NACIONAL CERRADO, 9., 2008, Brasília/DF. Anais... Brasília/DF: [s.n.], 2008, p. 1-3.

CARVALHO, G.D.; CAMPOS, A.B.; FAGERIA, N.K.; MADARI, B.E. Emissão de óxido nitroso derivada do cultivo de arroz irrigado em várzeas tropicais inundadas. Uberlândia/MG, 2011. In: CONGRESSO BRASILEIRO DE CIÊNCIA DO SOLO - CBCS, 33., 2011, Uberlândia/MG, Anais... Uberlândia: Universidade Federal de Uberlândia/ UFU, 2011, v. 1, p. 1-3.

CERRI, C.C. et al. Brazilian greenhouse gas emissions: the importance of agriculture and livestock. Scientia Agricola, v. 66, n. 6, p. 831-843, 2009.

CERRI, C.C.; CERRI, C.E.P. Agricultura e aquecimento global. Informativo da Sociedade Brasileira de Ciência do Solo, n. 23, p. 40-44, 2007.

CERRI, C.E.P.; SPAROVEK, G.; BERNOUX, M.; EASTERLING, W.E.; MELILLO, J.M.; CERRI, C.C. Tropical agriculture and global warming: Impacts and mitigation op- 
tions. Scient Agricult, n. 64, p. 83-99, 2007.

CHAVEZ, L. F. Balanço da emissão de gases de efeito estufa em Argissolo Vermelho sob sistemas de cultura em plantio direto. 2011. 120f. Tese (Doutorado) - Universidade Federal do Rio Grande do Sul, Faculdade de Agronomia, Porto Alegre, 2011.

CHIRINDA, N.; KRACHER, D.; L/EGDSMAND, M.; PORTER, J.R.; OLESEN, J.E.; PETERSEN, B.M.; DOLTRA, J.; KIESE, R.; BUTTERBACH-BAHL, K. Simulating soil N2O emissions and heterotrophic $\mathrm{CO} 2$ respiration in arable systems using FASSET and MoBiLE-DNDC. Plant Soil, n. 343, p.139-160, 2011.

CIAMPITTI, I.A.; CIARLO, E.A.; CONTI, M.E. Nitrous oxide emission during soybean culture: inoculation and nitrogen fertilization effects. Ciencia del Suelo, n. 23, p. 123-131, 2005.

COSTA, F.S.; GOMES, J.; BAYER, C.; MIELNICZUK, J. Métodos para avaliação das emissões de gases do efeito estufa no sistema solo-atmosfera. Revista Ciência Rural, v. 36, p. 693-700, 2006.

COUTINHO, R.P.; URQUIAGA, S.; BODDEY, R.M.; ALVES, B.J.R.; TORRES, A.Q.A.; JANTALIA, C.P. Estoque de carbono e nitrogênio e emissão de N2O em diferentes usos do solo na Mata Atlântica. Pesquisa Agropecuária Brasileira, v. 45, p. 195$203,2010$.

DALAL, R.C.; WANG, W.; ROBERTSON, G.P.; PARTON, W.J. Nitrous oxide emission from Australian agricultural lands and mitigation options: a review. Australian Journal of Soil Research, v. 41, p. 165-195, 2003.

DAVIDSON, E.A.; KELLER, M.; ERICKSON, H.E.; VERCHOT, L.V.; VELDKAMP, E. Testing a conceptual model of soil emissions of nitrous and nitric oxides. BioScience, v. 50, p. 667-680, 2000.

DENEGA, G.L. Emissão de óxido nitroso e dióxido de carbono após aplicação de dejetos de suínos e bovinos em um Argissolo. Santa Maria/RS. 2009. 92f. Dissertação em Agronomia (Mestre em Ciência do Solo) - Universidade Federal de Santa 
Maria/UFSM, Santa Maria, 2009.

DUXBURY, J.M. The significance of greenhouse gas from soils of tropical agroecosystems. In: LAL, R.; KIMBLE, J.; LEVINE, E.; STEWART, B. A. (Ed.) Soil management and greenhouse effect: advances in soil science. Boca Raton: CRC Lewis Publishers, 1995. p. 279-291.

ESCOBAR, L.F. Emissão de gases de efeito estufa em sistemas de manejo em solo do planalto médio do Rio Grande do Sul. 2008. 104f. Dissertação (Mestrado em Ciência do Solo) - Universidade Federal de Santa Maria/UFSM, Santa Maria, 2008.

FORSTER, P.; RAMASWAMY, V.; ARTAXO, P.; BERNTSEN, T.; BETTS, R.; FAHEY, D.W.; HAYWOOD, J.; LEAN, J.; LOWE, D.C.; MYHER, G.; NGANGA, J.; PRINN, R.; RAGA, G.; SCHULZ, M.; VAN DORLAND, R. Changes in atmospheric constituents and in radiative forcing. In: SOLOMON, S.; QUIN, D.; MANNING, M.; JOHNSON, J.M.F.; REICOSKY, D.C.; ALLMARAS, R.R.; SAUER, T.J.; VENTEREA, R.T.; DELL, C.J. Greenhouse gas contributions and mitigation potential of agriculture in the central USA. Soil e Tillage Research, v. 83, p. 73-94, 2005.

FROUZ, J.; PIZL, V.; CIENCIALA, E.; KALCIK, J. Carbon storage in post-mining forest, the role of tree biomass and soil bioturbation. Biogochemistry, v. 94, p. 111-121, 2009.

FRYE, W. Nitrification inhibition for nitrogen efficiency and environment protection. In: IFA INTERNATIONAL WORKSHOP ON ENHANCED:EFFICIENCY FERTILIZERS. Germany, v. 8, p. 28-30, 2005.

GOMES, J. Emissão de gases do efeito estufa e mitigação do potencial de aquecimento global por sistemas conservacionistas de manejo do solo. 2006. 126f. Tese (Doutorado em Ciência do Solo) - Universidade Federal do Rio Grande do Sul, Porto Alegre, 2006.

GOMES, J. et al. Soil nitrous oxide emissions in long-term cover crops: based rotations under subtropical climate. Soil \& Tillage Research, v. 106, n. 1, p. 36-44, 2009. 
GONÇALVES, C. E. A. Estudo da produção de óxido nitroso em cultivo de feijão (Phaseolus vulgaris). 2002. 91f. Dissertação (Mestrado em Geofísica Espacial) - Instituto Nacional de Pesquisas Espaciais/INPE, São José dos Campos, SP, 2002.

HARRISON, R.; WEBB, J. A review of the effect of $\mathrm{N}$ fertilizer type on gaseous emissions. Advances in Agronomy, v. 73, p. 65-108, 2001.

HATZENPICHLER, R. Diversity, Physiology and Niche Differentiation of Ammonia-Oxidizing Archaea. Applied and Environmental Microbiology, v. 78, n. 21, p.75017510, 2012.

INTERGOVERNMENTAL PANEL ON CLIMATE CHANGE. IPCC. Guidelines for National Greenhouse Gas Inventories. IPCC, UK, 2007. Vol. 4: Agriculture, Forestry and Other Land Use, Chapter 11, p. 5-6, S.

JANTALIA, C.P. et al. Em busca da mitigação da produção de óxido nitroso em sistemas agrícolas: Avaliação das práticas usadas na produção de grãos no Sul do Brasil. In: ALVES, B.J.R. et al. Manejo de sistemas agrícolas: impacto no sequestro de $\mathrm{C}$ e nas emissões de gases de efeito estufa. Brasília: Embrapa Informações Tecnológicas; Porto Alegre: Gênesis, 2006. 215p.

JOHNSON, J.M.F.; REICOSKY, D.C.; ALLMARAS, R.R.; SAUER, T.J.; VENTEREA, R.T.; DELL, C.J. Greenhouse gas contributions and mitigation potential of agriculture in the central USA. Soil e Tillage Research, n. 83, p. 73-94, 2005.

LEMKE, R.; SOLBERG, E.; IZAURRALDE, C.; NYBORH, M. Seasonal nitrous oxide emissions from agricultural soils in the Parkland region of the Canadian Prairie. 2004. Disponível em: < http://paridss.usask.ca/factbook/soilcrop/cesar1.html.> Acesso em: 11 jun. 2014.

LIU, X.J.; MOSIER, A.R.; HALVORSON, A.D.; REULE, C.A.; ZHANG, F.S. Dinitrogen and N2O emissions in arable soils: Effect of tillage, N source and soil moisture. Soil Biology e Biochemistry, n. 39, p. 2362-2370, 2007.

MAGIEIRO, E.C. Emissão e balanço de gases do efeito estufa em argissolo subtropical sob integração lavoura-pecuária com ovinos. 106f. 2013. Tese (Doutora- 
do) - Universidade Federal do Rio Grande do Sul, Porto Alegre, RS, 2013.

MAGIERO, E.C.; ASSMANN, J.M.; BAGATINI, T.; TOMAZI, M.; BAYER, C.; VIERO, F.; CARVALHO, P.C.F. Carbono e nitrogênio da biomassa microbiana, atividade de urease e emissão de $\mathrm{N}_{2} \mathrm{O}$ com aplicação de esterco e urina de ovinos. Uberlândia/ MG, 2011. In: CONGRESSO BRASILEIRO DE CIÊNCIA DO SOLO - CBCS, 33., 2011, Uberlândia/MG, Anais... Uberlândia: Universidade Federal de Uberlândia/UFU, 2011. v. 1, p. 1-3.

MINISTÉRIO DA CIÊNCIA, TECNOLOGIA E INOVAÇÃO. MCTI. Estimativas anuais de emissões de gases de efeito estufa no Brasil. Brasília, DF, 2013. Disponível em: < http://gvces.com.br/arquivos/177/EstimativasClima.pdf $>$. Acesso em: 12 jul. 2014.

MOREIRA, F. M. S.; SIQUEIRA, J. O. Microbiologia e bioquímica do solo. 2. ed. Lavras, MG: Ed. da UFLA, 2006. 729p.

OLIVEIRA, W.R.D.; CARVALHO, A.M.; SOUZA, K.W.; OLIVEIRA, A.D.; BRAGA, L.M.; PINHEIRO, L.A.; PASSOS, L.; PULROLNIK, K.; RAMOS, M.L.G. Emissão de $\mathrm{N}_{2} \mathrm{O}$ em solo cultivado com soja em sistemas de integração Lavoura-Pecuária-Floresta (iLPF) e integração lavoura-pecuária (iLP). In: FERTBIO, 2012, Maceió. Anais... Maceió: SBCS/UFAL, 2012.

PIMENTEL, L. G. Emissão de óxido nitroso do solo em sistemas de sucessão de culturas e sua relação com a qualidade do resíduo vegetal. 2014. 64f. Dissertação (Mestrado) - Universidade Federal do Rio Grande do Sul, Faculdade de Agronomia, Porto Alegre, RS, 2014.

PIVA, J.T.; DIECKNOW, J.; BAYER, C.; ZANATTA, J.A.; MORAES, A. Emissão de gases de efeito estufa em sistema de integração lavoura pecuária nos campos gerais do Paraná. Pato Branco/PR, 2009. In: SYNERGISMUS SCYENTIFIC, 2009. Pato Branco, PR. Anais... Pato Branco: Universidade Tecnológica Federal do Paraná/UTFPR, 2009.

SAMINÊZ, T.C.O. Efeito do sistema de cultivo, tensão da água, biomassa microbiana e temperatura do solo nos fluxos de $\mathrm{CH}_{4}$ e $\mathrm{N}_{2} \mathrm{O}$ em solos de cerrados. 1999. 99f. Dissertação (Mestrado), Universidade de Brasília, Brasília, DF, 1999. 
SIGNOR, D. Estoques de carbono e nitrogênio e emissóes de gases do efeito estufa em áreas de cana-de-açúcar na região de Piracicaba. 2010. 120f. Dissertação (Mestrado em Ciência dos Solos) - Universidade de São Paulo, USP, Piracicaba, SP, 2010.

SIGNOR, D.; CERRI, C.E.P. Nitrous oxide emissions in agricultural soils: a review. Pesquisa Agropecuária Tropical, v. 43, n. 3, p. 322-338, 2013.

SKIBA, U.; BALL, B. The effect of soil texture and soil drainage on emissions of nitric oxide and nitrous oxide. Soil Use and Management, n. 18, p. 56-60, 2002.

STEVENS, R.J.; LAUGHLIN, R.J. Measutement of nitrous oxide and nitrogen emissions from agricultural soils. Nutrient Cycling in Agroecosystems, Dordrecht, v. 52, p. 131-139, 1998.

TOMAZI, M.; SALTON, J.C.; FAVARIN, R.P.; RETORE, M.; SANTOS, D.C.; BAYER, C. Emissão de $\mathrm{N}_{2} \mathrm{O}$ proveniente de excretas de bovinos em sistema de integração lavoura- pecuária e pastagem permanente na região tropical do Cerrado brasileiro. In: CONGRESSO BRASILEIRO DE CIÊNCIA DO SOLO, 34., 2013, Florianópolis. Ciência do solo: para quê e para quem: Anais... Viçosa, MG: Sociedade Brasileira de Ciência do Solo, 2013.

VARENNES, A.A. In: Produtividade dos solos e ambiente. Portugal: Escolar, 2003. p. 163-180.

VARGAS, V.P.; CANTARELLA, H.; MARTINS, A.A.; SOARES, J.S.; SOUSA, R.M.; HACKBARTH, C.; ANDRADE, C.A. Dicianodiamida (DCD) diminui emissão de N2O de solo incubado com diferentes níveis de palha de cana-de-açúcar e $\mathrm{N}$ mineral. In: FERTBIO 2012, 2012, Maceió. Anais... Maceió: SBCS/UFAL, 2012.

VELTHOF, G. L.; KUIKMAN, P. J.; OENEMA, O. Nitrous oxide emission from soils amended with crop residues. Nutrient Cycling in Agroecosystems, v 62, p. 249$261,2002$.

WASSMANN, R.; VLEK, P.L.G. Mitigating greenhouse gas emissions from tropical agriculture: scope and research priorities. Environment, Development and Sustaina- 
bility, n. 6, p. 1-9, 2004.

YU, K.W.; WANG, Z.P.; VERMOESEN, A.; PATRICK, W.H.; VAN CLEEMPUT, O. Nitrous oxide and methane emissions from different soil suspensions: effect of soil redox status. Biology and Fertlity of Soils, v. 34, p. 25-30, 2001.

ZUMFT, W.G.; KRONECK, P.M.H. Respiratory Transformation of Nitrous Oxide (N2O) to Dinitrogen by Bacteria and Archaea. Advances in Microbial Physiology. v. 52, p. 107-227, 2006.

Recebido em: 04 de abril de 2014 Aceito em: 28 de setembro de 2014 